Preprints are preliminary reports that have not undergone peer review.

They should not be considered conclusive, used to inform clinical practice, or referenced by the media as validated information.

\title{
Intravitreal Injections and Face Masks: Endophthalmitis Risk Before and During the COVID-19 Pandemic
}

\section{Saeed Karimi}

Ophthalmic Research Center, Research Institute for Ophthalmology and Vision Science, Shahid Beheshti University of Medical Sciences, Tehran, Iran https://orcid.org/0000-0002-3231-8414

Amir Mohammadzadeh

Ophthalmic Research Center, Research Institute for Ophthalmology and Vision Science, Shahid Beheshti University of Medical Sciences, Tehran, Iran

Alireza Ramezani

Ophthalmic Epidemiology Research Center, Research Institute for Ophthalmology and Vision Science, Shahid Beheshti University of Medical Sciences, Tehran, Iran

\section{Iman Ansari}

Ophthalmic Research Center, Research Institute for Ophthalmology and Vision Science, Shahid Beheshti University of Medical Sciences, Tehran, Iran

\section{Hosein Nouri ( $\square$ Hosein.nouri.2018@gmail.com )}

Ophthalmic Research Center, Research Institute for Ophthalmology and Vision Science, Shahid Beheshti University of Medical Sciences, Tehran, Iran https://orcid.org/0000-0003-1808-0443

\section{Seyed-Hossein Abtahi}

Ophthalmic Research Center, Research Institute for Ophthalmology and Vision Science, Shahid Beheshti University of Medical Sciences, Tehran, Iran https://orcid.org/0000-0002-1459-6752

\section{Homayoun Nikkhah ( $\nabla$ h.nikkhah52@gmail.com )}

Ophthalmic Research Center, Research Institute for Ophthalmology and Vision Science, Shahid Beheshti University of Medical Sciences, Tehran, Iran https://orcid.org/0000-0002-2414-4661

\section{Research Article}

Keywords: COVID-19, Infection, Face Mask, Intravitreal injection, Endophthalmitis

Posted Date: November 30th, 2021

DOI: https://doi.org/10.21203/rs.3.rs-1124994/v1

License: () (1) This work is licensed under a Creative Commons Attribution 4.0 International License. Read Full License 


\section{Abstract}

In this retrospective, single-center study, the records of patients with acute endophthalmitis following intravitreal bevacizumab (IVB) injections during the pre-COVID and COVID-19 eras were reviewed and compared. A total of 28,085 IVB injections were performed during the pre-COVID era; nine eyes of nine patients developed acute post-IVB endophthalmitis in this era, giving an overall incidence of $0.032 \%$ (3.2 in 10000 injections). In the COVID era, a total of 10,717 IVB injections were performed; four eyes of four patients developed acute post IVB endophthalmitis in this era, giving an overall incidence of $0.037 \%$ (3.7 in 10000 injections). The incidences of post-IVB endophthalmitis during these two eras were not different (P-value $=0.779$ ). The BCVA of the eyes affected during the COVID era showed poor improvement back to baseline values, after three months, even with prompt and maximum treatment. Face masking protocols seem unlikely to increase the risk of post-IVB endophthalmitis.

\section{Introduction}

The importance of intravitreal injections, specially intravitreal anti-vascular endothelial growth factor (anti-VEGF) injections, has increased in recent years due to their widespread use in managing retinal diseases such as diabetic macular edema (DME), choroidal neovascularization (CNV), and age-related macular degeneration (AMD) (1). Along with its therapeutic benefits, the intravitreal delivery route carries the risk of some side effects and complications, including subconjunctival hemorrhage, uveitis, retinal tear or detachment, and endophthalmitis $(2,3)$. Endophthalmitis is a severe, sight-threatening complication of intravitreal injections; among its known risk factors are type 2 diabetes mellitus, blebs, blepharitis, etc. $(3,4)$. Observance of health protocols can significantly limit the incidence of post-intravitreal injection endophthalmitis $(3,5)$; alterations in the former may give rise to changes in the latter. The probable impacts of health protocol amendments during the COVID-19 pandemic -e.g., mask-wearing mandates for patients and operating room staff (5) and increasing application of hand disinfectant solutions in hospitals $(6,7)$ during the COVID-19 pandemic $(8,9)$ - on endophthalmitis incidence after intravitreal injection of anti-VEGF drugs are under investigation; the findings, however, have been inconsistent. While some have suggested an additional risk of endophthalmitis with patients wearing masks during the sessions -theoretically, through an upward direction of exhaled vapors towards the periocular area, increasing the risk of infectious complications -(10), others have found no increase in the overall risk of endophthalmitis, attributable to face mask use (11).

In the present study, we investigated the effect of these changes in health protocols during the COVID pandemic on the incidence of postintravitreal injections endophthalmitis and compared the endophthalmitis rate during the COVID pandemic with that during the preCOVID era. Intravitreal bevacizumab (IVB) is the most commonly used intravitreal anti-VEGF in the center where the study was conducted; thus, the term "IVB" will be used instead of intravitreal anti-VEGF injection throughout this paper.

\section{Methods}

In conducting the present retrospective, single-center cohort study, the electronic medical records of the Torfeh University Eye Hospital (Tehran, Iran), affiliated with the Shahid Beheshti University of Medical Sciences, were accessed and queried for all cases of acute endophthalmitis following IVB injections, performed during two distinct periods, i.e., March 1st, 2013 to October 31st, 2019, and March 1st, 2020 to April 1st, 2021. The former period is from now on referred to as the "pre-COVID era", and the latter as the "COVID era" constituting approximately 80 and 12 months, respectively.

The study protocol was approved by the local ethics committee and adhered to the tenets of the declaration of Helsinki. The records of all patients who had undergone IVB injection during those periods were extracted from the hospital data archives using the international classification of the disease-10 (ICD-10) codes. Subsequently, among those records, all cases with acute post-IVB endophthalmitis were identified and extracted using the ICD-10 code of endophthalmitis. The acquired information included basic demographic data, injection indications, treatments and outcomes, bacterial smear and culture results, best-corrected visual acuity scores (BCVA) before the injection, after the onset of endophthalmitis symptoms, and three months after the treatment.

Post-IVB endophthalmitis cases were excluded if I) their bevacizumab administration was part of another surgical procedure, II) had any other intraocular procedures during the last six weeks before the IVB injection, and III) their acute endophthalmitis was secondary to other causes such as trauma or post-cataract surgery. Acute endophthalmitis after IVB was defined as a progressive inflammation in the vitreous cavity with or without inflammation of the anterior chamber, occurring in six weeks after the injection. The diagnosis was confirmed by a vitreoretinal surgeon. 
All injections in the Torfeh University Eye hospital were performed in the operating room under strict aseptic techniques. All of the patients studied in the COVID era wore face masks. Before the procedure, physicians scrubbed their hands and wore face masks and sterile gloves. Topical tetracaine $0.5 \%$ drops were used to achieve local anesthesia. After cleaning the skin around the eye with a solution of povidone-iodine $10 \%$ and instilling a single drop of povidone-iodine $5 \%$ in the cul-de-sac, a sterile lid speculum was placed. The rubber covering of the bevacizumab vial was wiped with cotton soaked in $5 \%$ betadine; $1.25 \mathrm{mg}(0.05 \mathrm{ml})$ bevacizumab (Avastin $\AA_{\text {; }}$ Genentech, Inc., South San Francisco, CA, USA) was drawn into an insulin syringe for each injection. The needle was changed, and a 30-gauge needle was used for injection at 3-4 mm posterior to the limbus. Topical antibiotic eye drops were prescribed for three days after injection (12).

In the case of post IVB endophthalmitis, immediate anterior chamber and vitreous tap was performed for all patients, followed by intravitreal injection of vancomycin $(1 \mathrm{mg})$ and ceftazidime $(2.25 \mathrm{mg}$ ). Early standard three-port pars plana vitrectomy (PPV) was performed within 24 hours of diagnosis in all cases. Fortified antibiotic eye drops (vancomycin and ceftazidime) and systemic intravenous vancomycin and ceftazidime were initiated for all patients. Oral prednisolone $1 \mathrm{mg} / \mathrm{kg}$ was prescribed 24 hours after PPV and continued for ten days.

Finally, to investigate the effect of the pandemic-associated adjustments in health protocols on the incidence of this complication, data from the two periods, i.e., the Pre-COVID and the COVID eras, were compared and analyzed. Normal continuous variables were described as mean and standard deviation, and qualitative variables as frequency and percentage. Chi-square $\left(\mathrm{K}^{2}\right)$ test, Wilcoxon signed-rank test, and Kruskal-Wallis test were used to evaluate visual acuity changes in relation to other variables. Fisher's exact test was applied when comparing variables from the Pre-COVID era with those from the COVID era. A P-value of less than 0.05 was considered statistically significant. Data were gathered and analyzed using IBM SPSS v.23.0. for Windows.

\section{Results}

\subsection{Data from the pre-COVID era}

During the pre-COVID era, a total of 28,085 IVB injections were performed at the Torfeh University Eye Hospital. Nine eyes of nine patients developed acute post IVB endophthalmitis, giving an overall incidence of $0.032 \%$ (3.2 in 10000 injections). Patients' mean ( $\pm S D)$ age was 63.78 years $( \pm 13.8$; range: $44-89)$. Six patients $(66.7 \%)$ were female. The indications for IVB injections were DME in 4 eyes (44.5\%), vitreous hemorrhage due to PDR in 3 eyes (33.3\%), and neovascular AMD (nAMD) in 2 eyes (22.2\%). Considering all IVB injections $(28,085$ injections) the incidences of post-IVB endophthalmitis were $0.047 \%, 0.059 \%$, and $0.026 \%$ in PDR, nAMD, and DME patients respectively $(p>0.05)$, in the pre-COVID era. The mean $( \pm S D)$ time between IVB injections and endophthalmitis presentation was 2.77 days $( \pm 1.25$; range: $1-6)$ in the pre-COVID era. Table-1 presents detailed information on the nine patients who developed post-IVB endophthalmitis in the pre-COVID era.

\subsection{Data from the COVID era}

During the COVID era, 10,717 IVB injections were performed at the Torfeh University Eye Hospital. Four eyes of four patients developed acute post IVB endophthalmitis giving an overall incidence of $0.037 \%$ (3.7 in 10000 injections). The patients' mean ( \pm SD) age was 63.25 years ( \pm 6.5 ; range: $55-69)$. Among the four, only one was male. The indication for IVB injections was DME in three eyes (75\%) and AMD in one eye $(25 \%)$. The mean $( \pm S D)$ time between IVB injections and the endophthalmitis presentation was 2.75 days $( \pm 1.71$; range: $1-5)$. Table-2 shows detailed information on patients with post-IVB endophthalmitis in the COVID era. There was no significant difference in the incidence of endophthalmitis in pre-COVID and COVID eras $(0.032 \%$ vs. $0.037 \%$; $p=0.779)$.

\subsection{Culture results}

Among the nine endophthalmitis cases documented during the pre-COVID era, culture results were negative in six (66.7\%) and showed staphylococcus epidermidis growth in three (33.3\%); one eye developed phthisis bulbi (11.1\%). Culture results were negative for all four endophthalmitis cases in the COVID era.

\subsection{Visual acuity changes and treatment outcomes}

In the pre-COVID era, the mean ( \pm SD) baseline (i.e., before IVB injections) BCVA was $1.18 \pm 0.62$ logarithm of the minimum angle of resolution (logMAR). The mean ( \pm SD) BCVA had decreased to $2.5( \pm 0.42)$ logMAR upon presentation with endophthalmitis (change: 1.32 $\pm 0.69 \log M A R, P=0.008)$, and improved to $1.94( \pm 0.88) \log M A R$ three months after the treatment (change: $-0.56 \pm 0.65 \log M A R, P=$ 
0.035), i.e., prompt treatment of acute post-IVB endophthalmitis with immediate intravitreal antibiotics and early PPV caused significant improvement of BCVA.

In the COVID era, the mean $( \pm S D)$ baseline BCVA before IVB injections was $1.05( \pm 0.62) \log M A R$. The mean BCVA decreased to $2.2( \pm 0.8)$ logMAR upon presentation with endophthalmitis (change: $1.15 \pm 0.58 \log M A R, P=0.068)$, and changed to $0.97( \pm 0.51) \log M A R$ three months after the treatment (change: $-1.21 \pm 0.65 \log M A R, P=0.042)$.

During the pre-COVID era, the mean $( \pm S D)$ difference between the final and baseline BCVA values was $0.76( \pm 0.9) \log M A R(P=0.025)$. In other words, poor visual outcomes, compared to baseline visual acuity of the affected eyes, were achieved after three months, even with prompt initiation of maximum-potency treatment at the time of presentation. Regarding the cases occurring in the COVID era, however, the mean ( $\pm S D)$ difference between the final (after three months) and baseline BCVA was $-0.08( \pm 0.52) \log M A R(P=0.785)$, implying that a satisfactory visual recovery from the infection, with close-to-baseline BCVA values, is achievable with immediate initiation of highly potent therapies.

Of note, visual acuity outcomes three months after the treatment were not correlated with sex, age, or injection indication $(p>0.05)$. There was no endophthalmitis following intravitreal injection in patients referred to this center with retinal vein occlusions (RVO) in both eras studied.

\section{Discussion}

The present study determined a post-IVB endophthalmitis rate of $0.032 \%$ (3.2 in 10,000 injections) in the pre-COVID period era and $0.037 \%$ (3.7 in 10,000 injections) in the COVID era; our results are consistent with the ranges reported in previous studies (13).

Post-IVB endophthalmitis is a serious complication. Our study showed that prompt treatment with immediate intravitreal antibiotics and early PPV brought about statistically significant visual acuity improvements in patients with post-IVB endophthalmitis, both in the preCOVID period and in the pandemic era. However, three months after the treatment, the final visual acuity was not significantly more than the baseline. Rayess and colleagues found that the visual acuity in patients with endophthalmitis after anti-VEGF injections (Bevacizumab, Ranibizumab, and Aflibercept), at a 3-month follow-up, remained worse than that before the causative injection (14). Based on previous studies, factors affecting the development of endophthalmitis following IVB can be divided into clinical and technical. Clinical factors that can increase the risk of endophthalmitis include insulin-dependent diabetes mellitus (DM), older age, and blepharitis. DM and older age have also been associated with immunosuppression and increased susceptibility to infection $(14,15)$. Among technical factors is the type of surgical equipment used, as well as how well health protocols are observed.

Overall, we found that altered health protocols during the COVID pandemic had no statistically significant effect on the incidence of postIVB endophthalmitis. A few studies have evaluated the effect of universal face mask-wearing and other pandemic health protocols on the rate of endophthalmitis after intravitreal injections $(11,16)$. A multicenter and retrospective study has reported that universal face mask use during intravitreal injections did not increase the risk of developing presumed endophthalmitis, but it was associated with a lower rate of culture-positive endophthalmitis (17). In another study by Patel et al., it was shown that physician face mask use did not influence the risk of post-injection endophthalmitis compared to a no-talking policy (11). There is a hypothesis that facial mask fitting can effectively affect bacteria transmission and might affect the risk of post intravitreal injection endophthalmitis (18). In a study by Hadayer et al., it was emphasized that patients who wear face masks during intravitreal injections might be at a higher risk of endophthalmitis, which is why they recommended proper face mask fitting and either taping the upper edges of the face masks with a medical adhesive tape or using an adhesive surgical drape around the injected eye (19). Another study by simulation of intravitreal injections concluded that adding tape to the superior portion of the patient's face mask reduces bacterial dispersion during intravitreal injections. Also, there was no difference in bacterial dispersion compared with wearing N95 masks (20). Another study has suggested that securing the superior portion of the patient's face mask with tape may reduce bacterial dispersion or air particles toward the eye (21). However, it has been reported that this measure has no effect on endophthalmitis risk in patients undergoing IVB injections (17).

Some limitations apply to the present study. Given the retrospective nature of this study, potential errors in data registered in the hospital records could have been present; however, restrictive measures were taken to minimize such errors. With the ongoing pandemic, a decline in the number of patients -especially diabetic patients, many of whom suffer from other underlying comorbidities- referring to hospitals (studied by Al-Khersan et al. (22)) is a limitation that applies to many hospital-based studies; such limitation is more prominent in cohort studies evaluating the incidence of an uncommon complication, such as ours. Another limitation was that information on different modes of mask use, such as taping the upper edges or not, among our patients had not been documented. 


\section{Conclusion}

The present study showed that the incidence of post IVB endophthalmitis in the COVID era was not significantly different from that before the pandemic. Regardless of the pandemic-related alterations in health protocols adopted, endophthalmitis remains a rare complication after intravitreal injections; despite its rarity, post-IVB endophthalmitis has a poor prognosis and can lead to significant, and even complete, visual loss. The time interval between the IVB-injection and presentation of endophthalmitis is relatively short; prompt treatment with immediate intravitreal antibiotics and early pars plana vitrectomy are vital in maximizing the treatment outcomes.

\section{Declarations}

\section{Acknowledgments}

None

\section{Funding details}

No fundings were received for the conduction of this study.

\section{Disclosure of interest}

The authors report no conflict of interest

\section{Ethics approval}

This study was approved by the ethics committee of the Torfeh University Eye Hospital, Shahid Beheshti University of Medical Sciences.

\section{Consent to participate}

Not applicable.

\section{Consent for publication}

Not applicable.

\section{Data availability statement}

The data that support the findings of this study will be shared by the corresponding author, Homayoun Nikkhah, upon reasonable request.

\section{References}

1. Maloney MH, Payne SR, Herrin J, Sangaralingham LR, Shah ND, Barkmeier AJ. Risk of systemic adverse events after intravitreal bevacizumab, ranibizumab, and aflibercept in routine clinical practice. Ophthalmology. 2021;128(3):417-24.

2. Soliman MK, Gini G, Kuhn F, Iros M, Parolini B, Ozdek S, et al. International practice patterns for the management of acute postsurgical and postintravitreal injection endophthalmitis: European Vitreo-Retinal Society endophthalmitis study report 1. Ophthalmology Retina. 2019;3(6):461-7.

3. Lyall DA, Tey A, Foot B, Roxburgh ST, Virdi M, Robertson C, et al. Post-intravitreal anti-VEGF endophthalmitis in the United Kingdom: incidence, features, risk factors, and outcomes. Eye. 2012;26(12):1517-26.

4. Fintak DR, Shah GK, Blinder KJ, Regillo CD, Pollack J, Heier JS, et al. Incidence of endophthalmitis related to intravitreal injection of bevacizumab and ranibizumab. Retina. 2008;28(10):1395-9.

5. Klompas M, Morris CA, Sinclair J, Pearson M, Shenoy ES. Universal masking in hospitals in the Covid-19 era. New England Journal of Medicine. 2020;382(21):e63.

6. Al-Sayah MH. Chemical disinfectants of COVID-19: an overview. Journal of water and health. 2020;18(5):843-8.

7. Rabenau H, Kampf G, Cinatl J, Doerr HW. Efficacy of various disinfectants against SARS coronavirus. Journal of Hospital Infection. 2005;61(2):107-11. 
8. Advani SD, Smith BA, Lewis SS, Anderson DJ, Sexton DJ. Universal masking in hospitals in the COVID-19 era: Is it time to consider shielding? Infection Control \& Hospital Epidemiology. 2020;41(9):1066-7.

9. Wang X, Ferro EG, Zhou G, Hashimoto D, Bhatt DL. Association between universal masking in a health care system and SARS-CoV-2 positivity among health care workers. Jama. 2020;324(7):703-4.

10. Wen JC, McCannel CA, Mochon AB, Garner OB. Bacterial dispersal associated with speech in the setting of intravitreous injections. Archives of ophthalmology. 2011;129(12):1551-4.

11. Patel SN, Hsu J, Sivalingam MD, Chiang A, Kaiser RS, Mehta S, et al. The Impact of Physician Face Mask Use on Endophthalmitis After Intravitreal Anti-Vascular Endothelial Growth Factor Injections. American journal of ophthalmology. 2021;222:194-201.

12. Storey P, Dollin M, Pitcher J, Reddy S, Vojtko J, Vander J, et al. The role of topical antibiotic prophylaxis to prevent endophthalmitis after intravitreal injection. Ophthalmology. 2014;121(1):283-9.

13. Xu K, Chin EK, Bennett SR, Williams DF, Ryan EH, Dev S, et al. Endophthalmitis after intravitreal injection of vascular endothelial growth factor inhibitors: management and visual outcomes. Ophthalmology. 2018;125(8):1279-86.

14. Rayess N, Rahimy E, Storey P, Shah CP, Wolfe JD, Chen E, et al. Postinjection endophthalmitis rates and characteristics following intravitreal bevacizumab, ranibizumab, and aflibercept. American journal of ophthalmology. 2016;165:88-93.

15. Geerlings SE, Hoepelman AI. Immune dysfunction in patients with diabetes mellitus (DM). FEMS Immunology \& Medical Microbiology. 1999;26(3-4):259-65.

16. Patel SN, Tang PH, Storey PP, Wolfe JD, Fein J, Shah SP, et al. The Influence of Universal Face Mask Use on Endophthalmitis Risk after Intravitreal Anti-Vascular Endothelial Growth Factor Injections. Ophthalmology. 2021.

17. Patel SN, Tang PH, Storey PP, Wolfe JD, Fein J, Shah SP, et al. The influence of universal face mask use on endophthalmitis risk after intravitreal anti-VEGF injections during the COVID-19 pandemic. Ophthalmology. 2021.

18. Doshi RR, Leng T, Fung AE. Reducing oral flora contamination of intravitreal injections with face mask or silence. Retina. 2012;32(3):473-6.

19. Hadayer A, Zahavi A, Livny E, Gal-Or O, Gershoni A, Mimouni K, et al. Patients wearing face masks during intravitreal injections may be at a higher risk of endophthalmitis. Retina. 2020;40(9):1651-6.

20. Patel SN, Mahmoudzadeh R, Salabati M, Soares RR, Hinkle J, Hsu J, et al. Bacterial dispersion associated with various patient face mask designs during simulated intravitreal injections. American Journal of Ophthalmology. 2021;223:178-83.

21. Schultheis WG, Sharpe JE, Zhang Q, Patel SN, Kuriyan AE, Chiang A, et al. Effect of taping face masks on quantitative particle counts near the eye: Implications for intravitreal injections in the covid-19 era. American Journal of Ophthalmology. 2021;225:16671.

22. Al-Khersan H, Kalavar MA, Tanenbaum R, Lazzarini TA, Patel NA, Yannuzzi NA, et al. Emergent ophthalmic surgical care at a tertiary referral center during the COVID-19 pandemic. American journal of ophthalmology. 2021;222:368-72.

\section{Tables}

Table-1. Demographic, clinical, and culture information of patients with post-IVB endophthalmitis during the pre-COVID era 


\begin{tabular}{|c|c|c|c|c|c|c|c|c|c|c|c|}
\hline \multirow[t]{2}{*}{ ID } & \multirow[t]{2}{*}{ Sex } & \multirow[t]{2}{*}{ Age } & \multirow[t]{2}{*}{ Indication } & \multirow{2}{*}{$\begin{array}{l}\text { Days to } \\
\text { presentation }\end{array}$} & \multicolumn{2}{|c|}{ Pre Injection BCVA } & \multicolumn{2}{|c|}{ Presentation BCVA } & \multicolumn{2}{|c|}{ final BCVA } & \multirow[t]{2}{*}{ Culture } \\
\hline & & & & & Snellen & LogMAR & Snellen & LogMAR & Snellen & LogMAR & \\
\hline 1 & $\mathrm{~F}$ & 60 & PDR & 4 & $1 / 10$ & 1 & $\mathrm{HM}$ & 2.6 & CF1m & 1.79 & No Growth \\
\hline 2 & $\mathrm{M}$ & 79 & CNV & 1 & CF3m & 1.31 & LP & 2.7 & $\mathrm{HM}$ & 2.6 & No Growth \\
\hline 3 & $\mathrm{~F}$ & 86 & PDR & 6 & $2 / 10$ & 0.7 & NLP & 3 & NLP & 3 & $\begin{array}{l}\text { Staphyloccous } \\
\text { epidermidis }\end{array}$ \\
\hline 4 & $\mathrm{M}$ & 65 & DME & 2 & $3 / 10$ & 0.52 & CF1m & 1.79 & $2 / 10$ & 0.7 & No Growth \\
\hline 5 & $\mathrm{~F}$ & 44 & DME & 2 & CF2m & 1.48 & $\mathrm{HM}$ & 2.6 & CF2.5m & 1.39 & No Growth \\
\hline 6 & $\mathrm{~F}$ & 58 & CNV & 2 & $2 / 10$ & 0.7 & $\mathrm{HM}$ & 2.6 & $1 / 10$ & 1 & $\begin{array}{l}\text { Staphyloccous } \\
\text { epidermidis }\end{array}$ \\
\hline 7 & $\mathrm{~F}$ & 88 & DME & 2 & CF3m & 1.31 & CF1m & 1.79 & CF2.5m & 1.39 & No Growth \\
\hline 8 & $\mathrm{~F}$ & 54 & DME & 4 & $1 / 10$ & 1 & LP & 2.7 & NLP & 3 & $\begin{array}{l}\text { Staphyloccous } \\
\text { epidermidis }\end{array}$ \\
\hline 9 & M & 50 & PDR & 2 & $\mathrm{HM}$ & 2.6 & LP & 2.7 & HM & 2.6 & No Growth \\
\hline $\begin{array}{l}\mathrm{Me} \\
\mathrm{SD}\end{array}$ & & 63.7 & \pm 13.8 & $2.78 \pm 1.56$ & & $\begin{array}{l}1.18 \pm \\
0.62\end{array}$ & & $\begin{array}{l}2.5 \pm \\
0.42\end{array}$ & & $\begin{array}{l}1.94 \pm \\
0.88\end{array}$ & \\
\hline
\end{tabular}

Abbreviations: BCVA, best-corrected visual acuity; CF, Counting Finger; CNV, choroidal neovascularization; DME, diabetic macular edema; F, female; HM, Hand Motion; LP, Light Perception; M, Male; NLP, No Light Perception; PDR, proliferative diabetic retinopathy

Table 2. Demographic, clinical, and culture information of patients with post-IVB endophthalmitis during the COVID era

\begin{tabular}{|c|c|c|c|c|c|c|c|c|c|c|c|}
\hline \multirow[t]{2}{*}{ ID } & \multirow[t]{2}{*}{ Sex } & \multirow[t]{2}{*}{ Age } & \multirow[t]{2}{*}{ indication } & \multirow{2}{*}{$\begin{array}{l}\text { Days to } \\
\text { presentation }\end{array}$} & \multicolumn{2}{|c|}{ Pre Injection BCVA } & \multicolumn{2}{|c|}{ Presentation BCVA } & \multicolumn{2}{|c|}{ final BCVA } & \multirow[t]{2}{*}{ Culture } \\
\hline & & & & & Snellen & LogMAR & Snellen & LogMAR & Snellen & LogMAR & \\
\hline 1 & $\mathrm{~F}$ & 69 & DME & 1 & $4 / 10$ & 0.4 & $1 / 10$ & 1 & $4 / 10$ & 0.4 & $\begin{array}{l}\text { No } \\
\text { Growth }\end{array}$ \\
\hline 2 & $\mathrm{~F}$ & 55 & DME & 5 & CF1m & 1.79 & $\mathrm{HM}$ & 2.6 & CF2m & 1.48 & $\begin{array}{l}\text { No } \\
\text { Growth }\end{array}$ \\
\hline 3 & $\mathrm{~F}$ & 61 & DME & 2 & CF3m & 1.31 & $\mathrm{HM}$ & 2.6 & $2 / 10$ & 0.7 & $\begin{array}{l}\text { No } \\
\text { Growth }\end{array}$ \\
\hline 4 & M & 68 & AMD & 3 & $2 / 10$ & 0.7 & $\mathrm{HM}$ & 2.6 & CF3m & 1.31 & $\begin{array}{l}\text { No } \\
\text { Growth }\end{array}$ \\
\hline $\begin{array}{l}\mathrm{M} \\
\mathrm{SD}\end{array}$ & & 63.2 & $( \pm 6.5)$ & $2.75 \pm 1.71$ & & $\begin{array}{l}1.05 \pm \\
0.62\end{array}$ & & $2.20 \pm 0.8$ & & $\begin{array}{l}0.97 \pm \\
0.51\end{array}$ & \\
\hline
\end{tabular}

Abbreviations: AMD, age-related macular degeneration; BCVA, best-corrected visual acuity; CF, Counting Finger; DME, diabetic macular edema; F, female; M, Male 